\title{
Lung Nodule Detection and Classification by Using Convolutional Neural Network
}

\author{
${ }^{1}$ Ruchita Tekade, ${ }^{2}$ Prof. Dr. K. Rajeswari \\ ${ }^{1,2}$ Computer Department, Pimpri Chinchwad College of Engineering, Savitribai Phule Pune University, Pune, \\ Maharashtra \\ Email: ruchitatekade@gmail.com; kannan.rajeswari@pccoepune.org
}

Received: 09 ${ }^{\text {th }}$ July 2018, Accepted: $14^{\text {th }}$ August 2018, Published: $31^{\text {st }}$ August 2018

\begin{abstract}
According to the study of World Health Organization (WHO), lung cancer is leading cause of death among all types of cancers (L. A. Torre et al., 2015). Lung cancer detection in early stage is became easy with the help of several image processing and machine learning techniques. Artificial Neural Network is proven to be the best technique for medical imaging, especially in lung cancer diagnosis. For lung nodule detection Computer Tomography (CT) scan images are preferred by so many researchers. The lung CT scans are extracted from Lung Image Database Consortium and Image Database Resource Initiative (LIDC-IDRI) (Armato III et al., 2015) dataset. To detect lung nodules from CT scans, various image preprocessing techniques such as thresholding, clearing border and some morphological operations such as erosion, closing and opening are applied and after detecting Region of Interest (ROI), Convolutional Neural Network (CNN) is used to classify lung nodules using preprocessed images and it has given accuracy as $91.66 \%$ and sensitivity as $83 \%$.
\end{abstract}

Keywords: Machine Learning, Artificial Neural Network, Lung Nodule, Computer Tomography (CT), Lung Image Database Consortium and Image Database Resource Initiative (LIDC-IDRI), Thresholding, Clearing Border, Morphological Operations, Erosion, Closing, Opening, Convolutional Neural Network (CNN)

\section{Introduction}

In 2015, World Health Organization (WHO) has a survey for deaths and it was proved that cancer is second most leading cause of death (L. A. Torre et al., 2015). Lung cancer is most common cancer type which starts in lungs and can spread to other body parts. The human body has habit of checking and maintaining the growth of cells. Uncontrolled division of cells caused due to unbalanced system of growth of cells and it also forms mass. And this mass is nothing but tumor. Tumor can be produced in any body part and if it forms in lungs then it is known as lung nodules (I. Sluimer et al., 2006). Initially, lung nodules are very small in size (I. Sluimer et al., 2006).
Detection and classification of lung nodules at early stage is necessary in lung cancer diagnosis. Medical imaging is very useful in diagnosis of any kind of disease. Lung cancer diagnosis uses several kinds of images but CT scans are more preferred by previously implemented Computer Aided Diagnosis (CAD) (I. Sluimer et al., 2006) systems because it is more accurate (Armato III et al., 2015) and easy to process (Armato III et al., 2015). Recent studies have introduced different deep learning techniques for lung cancer diagnosis. Some of the studies are compared in literature review section. Most of the researchers have used LIDC-IDRI dataset (Armato III et al., 2015) to collect lung CT scan images. This dataset contains 1018 helical thoracic CT scans. Theses CT scans are taken from 1010 patients. These CT scan images are annotated by four trained radiologists. These annotation are according to the size of lung nodules and also the malignancy of cancer is given by these radiologists. This annotations are used to train the model. This study is divided into two phases: i) lung nodule detection also known as, Region of Interest (ROI) detection and ii) Lung nodule classification. ROI is the area where lung nodule like structure is present. For detection of ROI, some image processing techniques are applied on lung CT scan images as discussed further. These techniques includes thresholding, border clearing morphological erosion, morphological closing and morphological opening. After ROI detection, Convolutional Neural Network $(\mathrm{CNN})$ is applied on preprocessed images to classify lung nodules and non-nodules. CNNs plays very important and efficient role in medical imaging. These networks are very good at feature extraction and classification of the images. The results of preprocessing and classification are discussed in results and analysis section. Lung nodule classification using CNN after preprocessing gives $91.66 \%$ accuracy and $83 \%$ sensitivity.

\section{Literature Review}

In Multi group patch based CNN is proposed for better efficiency of lung nodule detection. In this study, two groups of image patches are used for classification. These lung CT scan images are preprocessed using highlighting lung regions with the help of local maximum variance, morphological operations 
including opening and closing, frangi filter (Hongyang Jiang et al., 2017), etc. After this preprocessing the lung nodule regions are cropped and one group is formed. And another group of patches is of original lung CT scan images. These original image patches group and preprocessed image patches group are provided to $\mathrm{CNN}$ for classification of lung nodules and non-nodules objects.

In, Multi -crop Convolutional Neural Network (MC$\mathrm{CNN}$ ) is proposed to automatically extract nodule salient information by employing a novel multi-crop pooling strategy which crops different regions from convolutional feature maps and then applies maxpooling different times. This approach automatically classifies nodule malignancy suspiciousness by extracting a set of highly compact features. A multicrop pooling operation (Wei Shen et al., 2016) is introduced which is a specialized pooling strategy for producing multi-scale features to surrogate the conventional max-pooling operation. Shuo Wang et al., 2017 have given a modified architecture of CNN is given for better efficiency rather than traditional CNN architecture. Typical CNN architecture has convolutional layer, pooling layer and fully connected layer with convolutional and pooling layer applied alternatively. The pooling layer is modified in this study using central pooling. In central pooling the way of applying max pooling is different than traditional pooling. In central pooling, the size of window varies for input space. The central pooling is applied row wise and column wise then max pooling is applied.

\section{Lung Nodule Detection}

After extracting lung CT scan images from LIDCIDRI dataset, the CT scans need to be preprocessed. CT scans may have some noise and distortion or unbalanced intensity distribution (i.e., low contrast). Lung CT scan objects such as lung contours or vessels should be removed lung nodule regions i.e., ROI should be detected (Choi W Jm et al., 2014). For these purposes, image preprocessing techniques are applied on CT scan images as shown in Fig. 1.

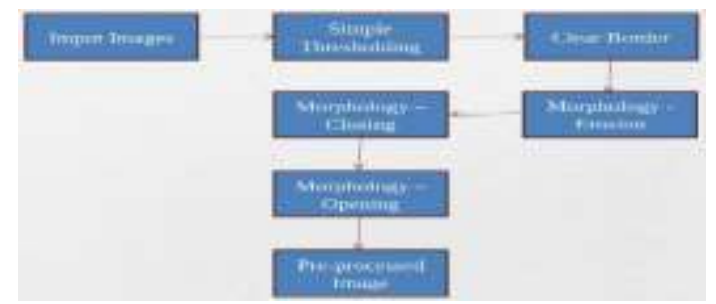

\section{Thresholding}

Fig. 1 Image Pre-Processing

Thresholding is well known and very simple technique which is used for converting grayscale images into binary images. The fix constant $\mathrm{T}$ is set initially as 127 and all pixel values in the image are get checked. If image pixel $I_{i, j}$ is less than $T\left(I_{i, j}<T\right)$, then $I_{i, j}$ is replaced by 0 . And if $\mathrm{I}_{\mathrm{i}, \mathrm{j}}$ is greater than or equal to $\mathrm{T}$ $\left(\mathrm{I}_{\mathrm{i}, \mathrm{j}}>=\mathrm{T}\right)$, then $\mathrm{I}_{\mathrm{i}, \mathrm{j}}$ is replaced by 255 (image pixels range from 0 to 255). So that binary image is formed having pixel values 0 or 255 .

\section{Border Clearing}

After thresholding, the objects are clearer to human eyes as well. But there are so many unwanted objects which are connected to the borders of the image. To remove such objects, clear border technique is used. It also suppresses the structures which have low intensity than its surroundings.

\section{Morphological Operations}

Morphology is another well-known technique in image processing to remove noise from the image which are created by simple thresholding. Morphological operations that are used in this study are: Erosion, Closing and Opening

Morphological erosion process the binary image $\mathrm{I}_{\text {in }}$ by using the kernel, also known as structuring element $\mathrm{K}$. The element $\mathrm{K}$ is placed on the image $\mathrm{I}_{\mathrm{in}}$ and if it fits on it the pixel is set as $1\left(\mathrm{I}_{\mathrm{out}}(\mathrm{x}, \mathrm{y})=1\right)$ otherwise, it is set as $0\left(\mathrm{I}_{\text {out }}(\mathrm{x}, \mathrm{y})=0\right)$. The erosion operation is denoted as, $I_{\text {out }}=I_{\text {in }} \ominus \mathrm{K}$. Erosion helps to increase gaps and holes between two objects and to reduce small details from image. Morphological dilation is opposite of erosion. It increases the size of bridge between two objects which are connected with very thin line. And morphological closing is the process dilation followed by erosion. It is denoted as, $\mathrm{I}_{\text {in }} \cdot \mathrm{K}$ where $\mathrm{I}_{\text {in }}$ is the input binary image and $\mathrm{K}$ is structuring element used in dilation and erosion. The holes between objects is get smaller and disruptions in the boundary of an object are filled. Morphological opening is the reverse process of closing. It is the process of erosion followed by dilation. Opening is denoted as $\mathrm{I}_{\text {in }} \mathrm{O} \mathrm{K}$, where $\mathrm{I}_{\text {in }}$ is input binary object and $\mathrm{K}$ in the structuring element used in erosion and dilation. This process helps to open up the gaps between objects connected with thin region. These techniques gives the preprocessed images from which lung nodules can be visualized by human eyes. After preprocessing the images are cropped to the objects which are visualized. These cropped images are also known as image patches (Marios Anthimopoulos et al., 2016). These patches are provided to $\mathrm{CNN}$ architecture for classification (Diaz JM et al., 2014).

\section{Lung Nodule Classification:}

Lung nodules (I. Sluimer et al., 2006) and non-nodules are detected in previous phase. Now two sets of lung nodules and non-nodules are created and provided to Convolutional Neural Network (CNN) (Kingsley 
Kuan et al., 2017) for training the model. CNN is the best way to extract the features and classify the images. CNN is nothing but the stack of multiple layers having different functionalities in both $2 \mathrm{D}$ and 3D CNN (Valente IRS et al., 2015) including:

1. Convolutional Layer

2. Polling Layer

3. ReLU Layer

4. Fully Connected layer

5. Loss Layer

\section{Convolutional Layer}

Convolutional layer is the one of the main building components in CNN architecture (Tianyi Liu et al., 2015). It is responsible for extracting the features from images (Jingjing Yuan et al., 2017). This layer uses the small matrix, also known as convolutional kernel $\mathrm{K}$ and it convolves throughout the input image I. The dot product of pixel of image with respect position value in kernel is taken and the sum of all dot products is placed in the output. The example of convolutional layer is shown on Fig. 2.

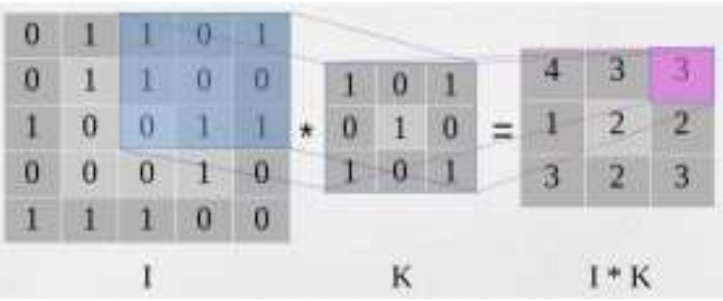

\section{Pooling Layer}

Fig. 2 Convolutional Layer

In pooling layer, only important features are extracted from convolved features. Pooling has two approaches as: max pooling and average pooling as shown in Fig. 3. Pooling uses $2 * 2$ window on features. In $\max$ pooling, only the maximum value among the 4 elements is included in output. And in average pooling the average of those 4 values is taken and placed in output. In this study max pooling of $2 * 2$ window is used. Every window in pooling, processes independently.

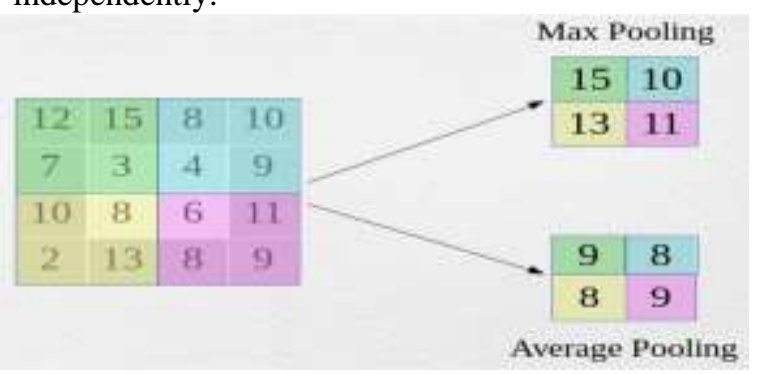

ReLU Layer

Fig. 3 Pooling Layer

The output of previous layers is in the range of -to + . And to understand if neuron is fired activation function is used to activate the neurons. Among the various types of activation functions like step function, tanh, sigmoid and ReLU, etc., ReLU is the most commonly used function. ReLU stands for Rectified Linear Unit. ReLU squash the input space from 0 to + . The function is defined as, $\mathrm{f}=\max (\mathrm{x}, 0)$. In other words, if the input value $\mathrm{x}$ is less than 0 then it is set as 0 or remain as it is otherwise. The working of ReLU function is shown in Fig. 4.

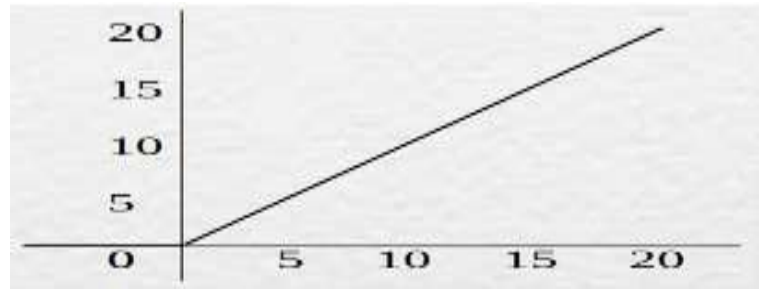

Fig. 4 ReLU Function

\section{Fully Connected Layer}

After feature extraction image classification is done at fully connected layer. It is also known as dense layer. In this layer, the features extracted by convolutional and pooling layer are get flattened in 1D array of neurons. Each and every neuron of fully connected layer is connected to each and every neuron of its previous layer. In this study, two dense layers are used with softmax function. Softmax function is used for classification of images. Softmax function computes the probabilities of image to be of either class. Whichever probability is more, image is assigned to that class. In this study, there are two types of classes, viz., nodules and non-nodules.

\section{Loss Layer}

This is last layer of $\mathrm{CNN}$ architecture and this layer includes loss function and optimizer. After classification, the loss is calculated using loss function, also known as error function or cost function. Loss of predicted probability is calculated according to the expected probability of classes. This loss is used to back propagate and update the weights until we reduce the loss as per the requirement. The loss function used in this study is, categorical cross entropy function. It is given as in equation (1),

$$
H(p, q)=-\sum p(x) \log (q(x))
$$

(Dr.Kevin Koidl et al., 2017) (1)

This function computes the entropy between the expected and predicted probabilities, here, $\mathrm{p}$ is the true probability distribution and $\mathrm{q}$ is the predicted distribution.

The optimizer helps to find accurate and optimal weights for CNN which can reduce the loss. There are various types of optimizers but we have used adam as 
it is more efficient than other optimizers. Adam is nothing but adaptive moment estimation. It is the combination of AdaGrad and RMSProp optimizers. The advantages of both the optimizers are combined in adam. Adam is suitable for the problem which has noisy and large data. The image patches created in lung nodule detection phase are provided to this CNN architecture (Tianyi Liu et al., 2015) and it gives the classification of lung nodules and non-nodules.

\section{Results and Discussion}

As discussed in previous sections, the image processing techniques are applied on the lung CT scan images for preprocessing as shown in Fig. 5.
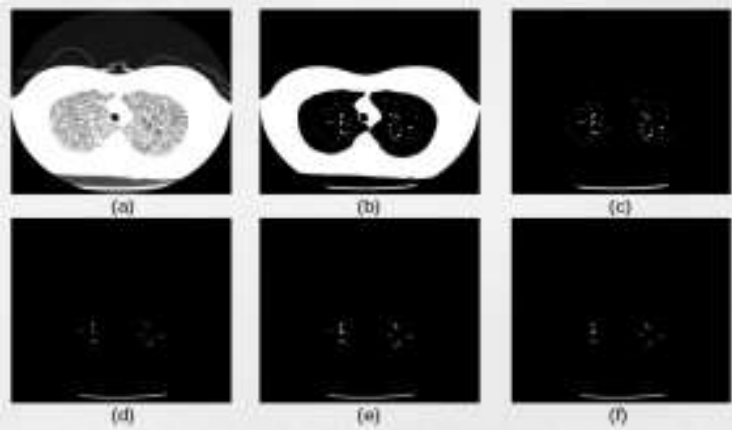

Fig. 5 Lung Nodule Detection

In this figure, (a) is the original lung CT scan image extracted from dataset. It has so many objects other than nodules. (b) is the output after applying simple thresholding on CT scan. Only important objects are highlighted and others are excluded. (c) is the output of border clearing technique. The lung contour connected to the border is removed. (d) is the output after applying morphological erosion on binary image. The size of object is reduced and unknown connections of 3D structure (Valente IRS et al., 2015) is removed. (e) is the output of morphological closing and (f) is the output of opening or filling. This gives the nodules and non-nodules structures. After detecting the nodules the image is cropped to the objects and two sets of nodules and non-nodules are prepared as shown in Fig. 6 and are provided to CNN for training with the annotations given in dataset. And then prediction can be done according to the labels as nodules and non-nodules.
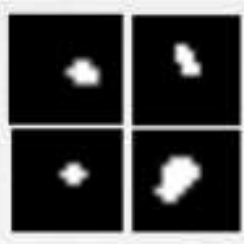

Nodules
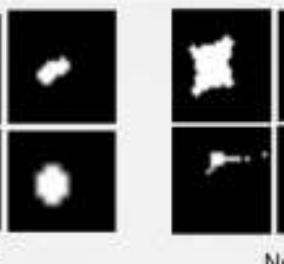

Non-Nodules

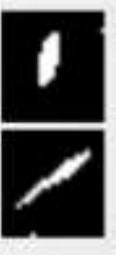

les
Fig. 6 Nodules and Non-nodules

The performance metrics used in this study is classification accuracy and sensitivity as given in equation (2) and (3) respectively,

Accuracy $=\frac{T P+T N}{(T P+F P+F N+T N)}$

...(Wen Zhu et al., 2010) (2)

$$
\text { Sensitivity }=\frac{T P}{T P+F N}
$$

...(Wen Zhu et al., 2010) (3)

The accuracy of CNN classification after applying preprocessing is $91.66 \%$ and Sensitivity is $83.33 \%$. The comparison of this study with previous work is shown in Table 1.

\begin{tabular}{|l|l|l|}
\hline Researches & No. of Samples & Sensitivity \\
\hline $\begin{array}{l}\text { Hongyang Jiang, et } \\
\text { al. }\end{array}$ & 1006 & 0.94 \\
\hline Wei Shen, et al. & 1375 & 0.77 \\
\hline Shuo Wang, et al. & 893 & 0.92 \\
\hline This work & 114 & 0.83 \\
\hline
\end{tabular}

Table 1: Results indicating sensitivity

\section{Conclusion}

The purpose of conducting this study is to analyze the effectiveness of convolutional neural network (CNN) in lung nodule detection and classification. By going through various research papers it is discovered that neural networks has played an very important role for feature extraction and classification rather than other traditional approaches of image classification. Preprocessing of images intend to better accuracy instead of providing whole image (Hongyang Jiang et al., 2017), as it reduce false positive rate. We have performed feature extraction and classification of lung nodules with $91.66 \%$ accuracy. The future work will be to classify the lung nodules into benign and malignant forms (Tizita Nesibu Shewaye et al., 2016) to detect the malignancy level of cancer in early stage and survival rate can be increased.

\section{References}

1. L. A. Torre, F. Bray, R. L. Siegel, J. Ferlay, J. Lorter Tieulent, and A. Jemal, "Global cancer statistics, 2012”, CA Cancer J Clin., vol. 65, no. 2, pp. 87-108, 2015.

2. I. Sluimer, A. Schilham, M. Prokop, and B. Ginneken, "Computer analysis of computed tomography scans of the lung: a survey", IEEE Trans. Med. Imaging, vol. 25, no. 4, pp. 385-405, 2006.

3. Armato III, Samuel G., McLennan, Geoffrey, Bidaut, Luc, McNitt-Gray, Michael F., Meyer, Charles R., Reeves, Anthony P., . . . Clarke, Lau-rence P. 
(2015). Data From LIDC-IDRI. The Cancer Imaging Archive. http://doi.org/10.7937/K9/TCIA.2015.LO9QL9SX. 4. Hongyang Jiang, He Ma, Wei Qian, Mengdi Gao and Yan Li, "An Automatic Detection System of Lung Nodule Based on Multi-Group Patch-Based Deep Learning Network", IEEE Journal of Biomedical and Health Informatics, 2017.

5. Wei Shen, Mu Zhou, Feng Yang, Dongdong Yu, Di Dong, Caiyun Yang, Yali Zang, Jie Tian, "Multi-crop Convolutional Neural Networks for lung nodule malignancy suspiciousness classification", Elsevier, Pattern Recognition 2016.

6. Shuo Wang, Mu Zhou, Zaiyi Liu, Zhenyu Liu, Dongsheng Gu, Yali Zang, Di Dong, Olivier Gevaert, Jie Tian, "Central focused convolutional neural networks: Developing a data-driven model for lung nodule segmentation", Elsevier B.V., Medical Image Analysis (2017) 172-183.

7. Marios Anthimopoulos, Stergios Christodoulidis, Lukas Ebner, Andreas Christe, Stavroula Mougiakakou, "Lung Pattern Classification for Interstitial Lung Diseases Using a Deep Convolutional Neural Network", IEEE Transactions on Medical Imaging, 2016.

8. Kingsley Kuan, Mathieu Ravaut, Gaurav Manek, Huiling Chen, Jie Lin, Babar Nazir, Cen Chen, Tse Chiang Howe, Zeng Zeng, Vijay Chandrasekhar, "Deep Learning for Lung Cancer Detection: Tackling the Kaggle Data Science Bowl 2017 Challenge", arXiv:1705.09435v1 [cs.CV] 26 May 2017.

9. Valente IRS, Cortez PC, Neto EC, Soares JM, de Albuquerque VHC, Tavares JMRS, "Automatic 3D pulmonary nodule detection in CT images: a survey", Computer Methods Programs Biomed. 2015;124:91107.

10. Tianyi Liu, Shuangsang Fang, Yuehui Zhao, Peng Wang, Jun Zhang, "Implementation of Training Convolutional Neural Networks", arXiv:1506.01195, 2015.

11. Dr. Kevin Koidl, "Loss Functions in Classification Tasks", School of Computer Science and Statistic Trinity College Dublin ADAPT Research Centre, 2017

12. Diaz JM, Pinon RC, Solano G., "Lung cancer classification using genetic algorithm to optimize prediction models", IISA 2014, 5th Int. Conf. Information, Intell. Syst. Appl. Chania: IEEE; 2014: $1-6$.
13. Choi WJ, Choi TS., "Automated pulmonary nodule detection based on three dimensional shapebased feature descriptor", Computer Methods Programs Biomed. 2014;113:37-54.

14. Tizita Nesibu Shewaye, Alhayat Ali Mekonnen, "Benign-Malignant Lung Nodule Classification with Geometric and Appearance Histogram Features", arXiv:1605.08350v1 [cs.CV], 2016.

15. Wen Zhu, Nancy Zeng, Ning Wang, "Sensitivity, Specificity, Accuracy, Associated Confidence Interval and ROC Analysis with Practical SAS® Implementations", Health Care and Life Sciences, 2010 